\title{
Realization of Solar Servo Control System for Vertical Aquarium
}

\author{
Hyeok Jae Woo ${ }^{1}$, Yogendra Rao Musunuri ${ }^{2}$ and Kyoo Jae Shin $^{3}$ \\ ${ }^{1,2,3}$ Department of ICT Creative Design, Graduate School, Busan University of \\ Foreign Studies, BUFS, Busan, Republic of Korea \\ ${ }^{1}$ reedvil@gmail.com, ${ }^{2}$ musunuri3@gmail.com, ${ }^{3}$ kyoojae@bufs.ac.kr
}

\begin{abstract}
In this paper, solar energy is a kind of clean energy for environment. Solar energy is rapidly gaining notoriety as an important means of expanding renewable energy resources. The solar servo control system designed for the vertical aquarium and solar tracking allows more energy to be produced because the solar array is able to remain aligned to the Sun. Hence it is the challenge for the present generation to generate maximum electricity by using solar power although many developments were made to collect the solar energy, but unable to utilize the maximum power released from sun. Hence solar tracking system has been implemented that plays a vital role in collecting the solar energy. The one axis solar tracking system produces sufficient energy for the vertical aquarium. The sun radiation falls on the photovoltaic cells and converts into the electrical energy. The main power generated from one axis solar system utilizes the Heat pump in the vertical aquarium. The efficiency of this system can be improved by the 5\% to $10 \%$.
\end{abstract}

Keywords: solar tracking system, solar azimuth, high driving torque, induction motor, vertical aquarium, Heat pump

\section{Introduction}

The solar energy is a renewable energy and it is important for providing the electricity. The Photovoltaic systems convert solar radiation to electricity via a variety of methods. The most common approach is to use silicon panels, which generate an electrical current when light shines upon it [1,2]. In this paper, solar servo control tracking device which consists of remotely controlling and monitoring a solar module assembly for smooth driven according to the solar azimuth. The solar azimuth is obtained from the following azimuth equation by using time $\mathrm{H}$ and declination. In this photovoltaic method is used to convert the sun light into the electricity. The solar tracking system provides a remotely servo controlling and monitoring the solar module assembly according to the solar azimuth measured by single solar cell sensor and solar module assembly that consists of solar panels arranged in $4 * 4$ matrix form to achieve high electric power generation with less cost $[1,3,4]$. I

The solar servo control tracking device also comprises of:

a). Integrated control device that consist single solar cell sensor unit detects the luminance of sunrays according to the solar azimuth.

b). Control panel that transmits control signals remotely with respect to maximum solar azimuth calculated by comparing with sunrays incident on the solar cell sensor unit and solar azimuth measured in real time.

c). Solar tracking device that includes a solar tracking device controller remotely that receives the control signals through wireless network.

d). High torque driving unit driven by AC single phase inductor to generate high torque with respect to the control signal. 
e). The operating angle of sensor unit is installed to high torque driving unit, to track the sun according to the control signal.

In this paper, the one axis solar tracking system is used to generate the power and send to the vertical aquarium.

\section{Modeling of Solar Servo Control System for Vertical Aquarium}

The Model consists of one axis solar tracking system, irradiation sensor, power house, waste water tank, heat pump, buffer tank and vertical aquarium building. The vertical aquarium using solar tracking system as shown in the Figure 1(a). The flow chart of this model has shown in the Figure 1(b). When the solar light fall on the one axis solar panel, the light sensor sense the solar radiation of the photo voltaic cell and send to the AC induction motor. Then, the AC motor can rotate the solar panel according to the sensor data and it converts the solar light into electrical energy and sends it into the power house to store and send to the heat pump and vertical aquarium.

\subsection{Heat Pump}

Heat pumps offer an energy-efficient alternative to furnaces and air conditioners. Heat flows naturally from a lower to higher temperature. Heat pumps, however, are able to force the heat flow in the other direction, using a relatively small amount of high quality drive energy (electricity, fuel, or high-temperature waste heat). Thus, heat pumps can transfer heat from natural heat sources, to a building vertical aquarium. Heat pumps can also be used for cooling. The heat pump in general can be operated in two modes namely heating mode and cooling mode. In the heating mode, the outdoor coil is an evaporator, while the indoor coil is a condenser. The refrigerant flowing from the evaporator (outdoor coil) carries the thermal energy from outside air to indoors, after the vapour temperature has been augmented by compressing it. The indoor coil then transfers thermal energy (including energy from the compression) to the indoor air, which is then moved around the inside of the building by an air handler. In cooling mode the cycle is similar, but the outdoor coil is now the condenser and the indoor coil (which reaches a lower temperature) is the evaporator. This is the familiar mode in which air conditioners operate.

\subsection{Vertical Aquarium}

The one axis solar tracking system is designed for the building vertical aquarium. The solar tracking system has one axis. This system mainly takes the solar energy and it can be converted into the electrical energy. This generated power will be send to the building vertical aquarium. The building vertical aquarium using one axis solar tracking system as shown in the Figure 1(a). 



Figure 1. (a) Building Vertical Aquarium using one Axis Solar Tracking System (b) Flow chart of the Solar Tracking System

When the solar light fall on the one axis solar panel, the maximum solar radiation absorbed photovoltaic cell measured by the irradiation sensor and converts it into electrical energy. The system consists of one axis solar panel, two axes solar tracking device, irradiation sensor and vertical aquarium building. The generated power utilizes entire vertical aquarium. The Vertical aquarium is specially designed for growing eels as fresh like in the sea water. The aquarium is the multi stairs, well electronically monitoring system. For this, one of the power cables from the one axis solar tracking system is connected to the vertical aquarium. Then, it takes power from the solar tracking system and utilizes for automatically monitored system.

\subsection{Modelling Equations of an Induction Motor for Solar Tracking System}

The two-phase induction motor is composed of two asymmetrical windings. Therefore, the auxiliary winding usually has fewer turns than the main winding and is displaced at ninety electrical degrees between these winding [6] [7] [8]. The auxiliary ( $\alpha)$ windings and main $(\beta)$ windings are not identical sinusoidal distributed windings, but are arranged in space quadrature. The equivalent circuits representing the asymmetrical two-phase induction motor in stationary $(\alpha \beta)$ reference frame. The dynamic model equation of asymmetrical two phase induction motor can be written as $\alpha \beta$ reference frame variables. Components stator and rotor voltage of the two-phase induction motor can be expressed as follows:

$$
\begin{gathered}
v_{s \alpha}=R_{s \alpha} i_{s \alpha}+\frac{d}{d t} \psi_{s \alpha} \\
v_{s \beta}=R_{s \beta} i_{s \beta}+\frac{d}{d t} \psi_{s \beta} \\
v_{r \alpha}=0=R_{r \alpha} i_{r \alpha}+\frac{d}{d t} \psi_{r \alpha}+a \omega_{r} \psi_{r \beta} \\
v_{r \beta}=0=R_{r \beta} i_{r \beta}+\frac{d}{d t} \psi_{r \beta}-\frac{1}{a} \omega_{r} \psi_{r \alpha}
\end{gathered}
$$


The components of stator and rotor flux linkages equations can also be expressed in the following equations:

$$
\begin{aligned}
& \psi_{s \alpha}=L_{s \alpha} i_{s \alpha}+L_{m \alpha} i_{r \alpha} \\
& \psi_{s \beta}=L_{s \beta} i_{s \beta}+L_{m \beta} i_{r \beta} \\
& \psi_{r \alpha}=L_{m \alpha} i_{s \alpha}+L_{r \alpha} i_{r \alpha} \\
& \psi_{r \beta}=L_{m \beta} i_{s \beta}+L_{r \beta} i_{r \beta}
\end{aligned}
$$

Using equation (5)-(8), as for the stator and rotor currents equations are given by:

$$
\begin{gathered}
i_{s \alpha}=\frac{L_{r \alpha} \psi_{s \alpha}-L_{m \alpha} \psi_{r \alpha}}{L_{s \alpha} L_{r \alpha}-L_{m \alpha}^{2}} \\
i_{r \beta}=\frac{L_{r \beta} \psi_{s \beta}-L_{m \beta} \psi_{r \beta}}{L_{s \beta} L_{r \beta}-L_{m \beta}^{2}} \\
i_{r \alpha}=\frac{L_{s \alpha} \psi_{r \alpha}-L_{m \alpha} \psi_{s \alpha}}{L_{s \alpha} L_{r \alpha}-L_{m \alpha}^{2}} \\
i_{r \beta}=\frac{L_{s \beta} \psi_{r \beta}-L_{m \beta} \psi_{s \beta}}{L_{s \beta} L_{r \beta}-L_{m \beta}^{2}}
\end{gathered}
$$

The equation of electromagnetic torque produced by the machine is then given by the equation:

$$
T_{e}=p_{p}\left(L_{m \beta} i_{s \beta} i_{r \alpha}-L_{m \alpha} i_{s \alpha} i_{r \beta}\right)
$$

and the mechanical dynamic is modeled by the equation.

$$
J \frac{d}{d t} \omega_{r}=T_{e}-T_{L}
$$

\section{Construction Details and Working}

The 1 axis solar tracking unit mainly consists of two unit's namely remote total controller and tracker controller as shown in the Figure 2. The remote total controller consists of PID controller and 1 axis absolute angle output unit. The tracker controller has PID angle controller, 1 axis driver circuit and 1 axis tracker for AC motor. When the sun light as giving the input to the PID controller, it sends the control signal to the 1 axis absolute angle unit. The 1 axis absolute angle 




\section{Figure 2. The 1 Axis Solar Tracking Unit (A) 6 Section Reduce Solar Tracking Device, (B) Integrated Remote Control Panel, (C) Ambient Light Sensor, (D) Integrated Control Panel, (E) Board, (F) Speed Driving Unit}

unit sends the absolute angle to the PID angle controller and it sends the signal to 1 axis driver circuit. The 1 axis driver circuit sends the signal and rotates the 1 axis tracker. Then, it will be fine-tuned to maximum intensity of solar radiation and give the electrical voltage as output. When any disturbance comes, the signal will be feed backed to the PID angle controller. The block diagram of one axis solar tracking unit as shown in the Figure 3. Each solar module assemblies consists of $4 * 4$ matrix array solar panel, number of solar modules are $6(1,2,3,4,5,6)$ are shown in the Figure $4(a)$. The arrangement of solar plates as shown in the Figure 4(b). These modules weight is $20 \%$ more than the existing modules to increase the photovoltaic power generation by high driving torque unit to track the sun rays. The high driving torque is installed in between third and fourth solar module assemblies and fixed to the adjusting shaft, to drive the six module assemblies at once. The operating angles of solar module assemblies and operation states of AC single phase inductor are remotely monitored. Adjusting that is supported by A-shaped support and fixed to the concrete base as shown in Figure 4(c). The 1 to 6 solar module assemblies can be driven with operating angle range of 30 degrees to 150 degrees with tracking device controller; the angles are detected by operating angle sensors are as shown in the Figure 5, that are fixed to the driving torque.

The driving torque unit consists of cooling fans attached to the AC single phase inductor which are driven through the servo control that can be done by tracking device controllers that receives the control signal from the control panel (500). The mechanism in the driving torque is as follows, the pinion of third reducer is connected to secondary reducer, and the worm wheel is connected to the pinion to generate maximum torque. The adjusting shaft is fixed on the worm wheel. The driving force of the pinion of third worm reducer is transmitted to worm wheel at a gear ratio of 55:1; worm wheel is meshed with power transmission rotary shaft as shown in the Figure 6(a). 


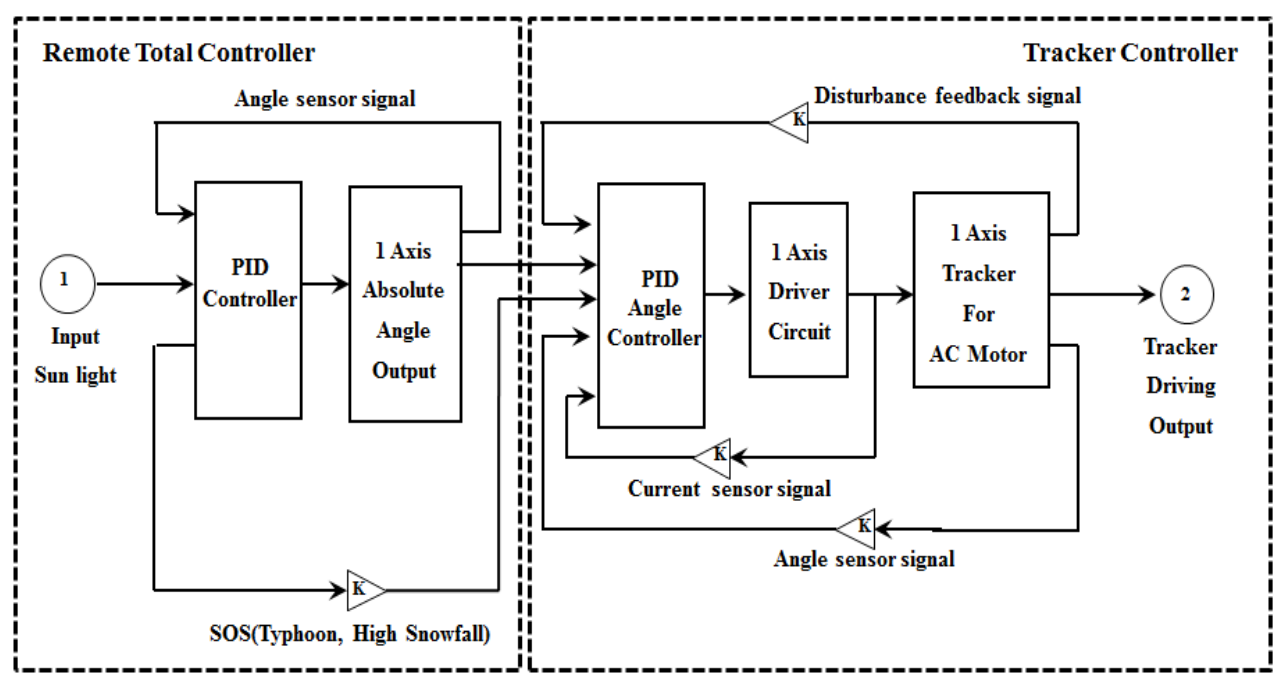

Figure 3. The Block Diagram of 1 Axis Solar Tracking Unit.

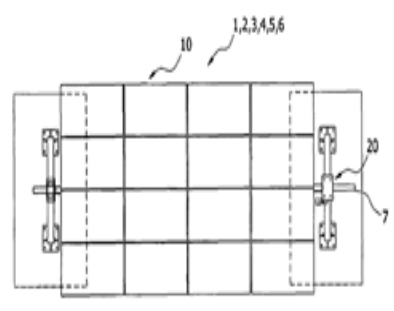

(a)

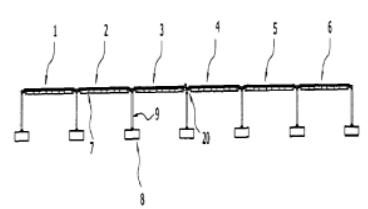

(b)

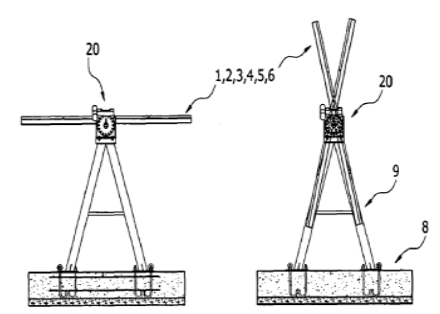

(c)

Figure 4. (A) Arrangement of Solar Module Assembly, (B) Solar Plates Arrangement in a Row and (C) Solar Servo Tracking Device with A-Shaped Support

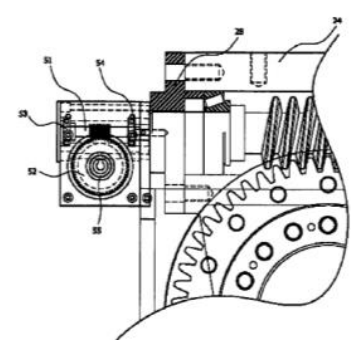

(a)

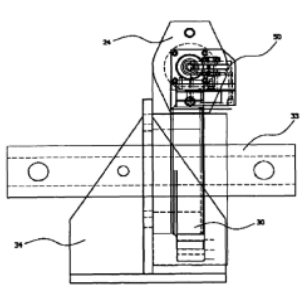

(b)



(c)

Figure 5. Operating Angle Sensor Unit.(A) Right Side View of A Power Driving Unit of the Solar Servo Control Tracking Device, (B) Left Side View of the Power Driving Unit of the Solar Servo Control Tracking Device,(C) An

Enlarged View of Illustrating the Operating Angle Sensor Unit 


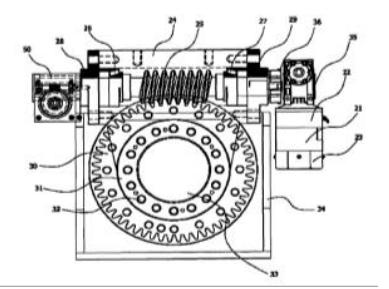

(a)

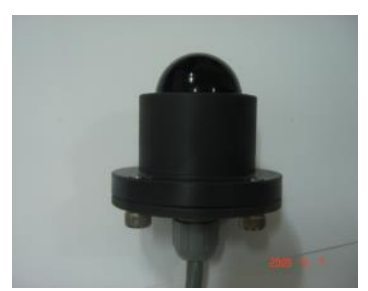

(b)



(c)

Figure 6. (A) Front View Illustrating Structure of Power Transmission of Solar Servo Control Tracking Device, (B) Illustrating An Actual Appearance of An Operating Angle Sensor Unit and (C) One Axis Irradiation Sensor

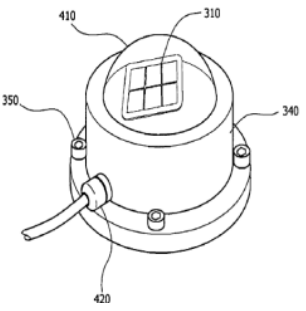

(a)

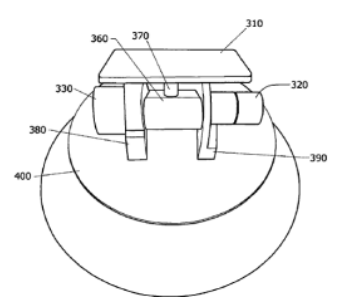

(b)

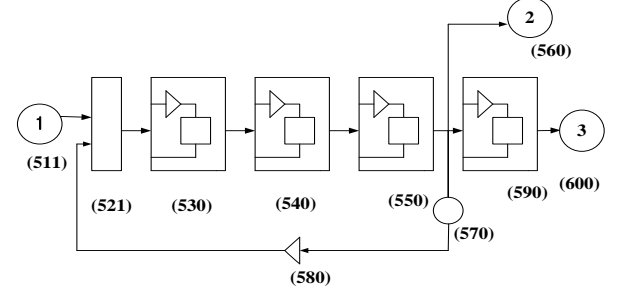

(c)

Figure 7. Solar Cell Sensor Unit According To Present Invention, (A) Illustrating an Actual Appearance of Solar Cell Sensor Unit (B) Illustrating An Actual Structure of Solar Cell Sensor Unit (C) Schematic Diagram of Measurement of Solar Azimuth By Integrated Control Panel

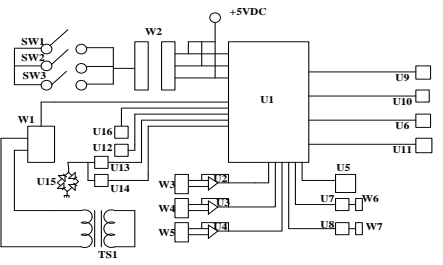

(a)

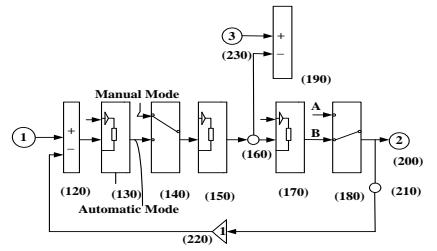

(b)

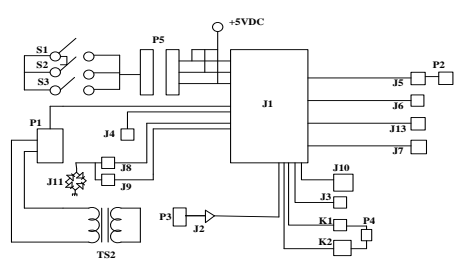

(c)

Figure 8 Schematic Diagram of Operating Angle Servo Control of Solar Module Assembly,(A) Illustrating The Integrated Control Panel of the Solar Servo Control Tracking Device(B) Illustrating An Operating Angle Control Panel of the Solar Servo Control Tracking Device(C) Illustrating A Tracking Device Controller of Solar Servo Tracking Device.

The mechanism for servo control of rotation angle of solar module assemblies are as follows (Figure 6(b)),as sensor pinion wheel meshed with pinion of third reducer that transmits the gear ratio to the sensor wheel, the operating angle sensor used to detect 360 degrees is installed on a rotation shaft on the sensor worm wheel to detect absolute rotation angle. From the sensor's output voltage, the console calculates and displays solar irradiance. It also integrates the irradiance values and displays total incident energy over a set period of time. The one axis irradiation sensor as shown in the Figure 6(c). The solar cell sensor unit mechanism: the DC motor provides rotary power to measure incident angle of sun rays that is entering the solar cell up to 30 degrees to 150 degrees [8]. The Figure 7 shows the solar cell sensor unit 300 measures luminance of sunrays at a solar azimuth to detect a maximal solar azimuth of the sunrays entering at a maximal incident angle. A solar cell rotation control signal 511 of 30 degrees to 150 degrees is periodically input to the solar cell sensor unit 300. A rotation angle calculator 521 compares a rotation 
angle signal 580 detected and amplified by a rotation angle sensor 570 with the solar cell rotation angle control signal 511. An error of the solar cell rotation angle is compensated by a proportional-integral-derivative (PID) servo controller 530 and is applied to a stepping motor driver 540.The stepping motor driver 540 drives the stepping motor 550 and in this case the rotation angle 560 is detected by the rotation angle sensor 570 to be fed back to the rotation angle calculator 521. A rotation angle of a solar cell 590 fixed to the stepping motor 550 is controlled by the stepping motor 550 and continuously scans luminance 600 every rotation angle of the solar cell 590 so that maximal luminance of the sunrays with respect to the rotation angle can be detected and shown in the Figure 5(c).

The circuit diagrams of the integrated control panel 500 detecting the maximal luminance as depicted in Figure 6(a) and the integrated control panel 500 includes a manual/ automatic mode switch SW1 selected by an operator, a real time counter (DS1307) U5 to which a period is set to measure the solar azimuth by time period in real time, a first buffer circuit $\mathrm{U} 2$ to Which a rotation angle of the solar cell sensor unit 300 is input through the manual/automatic mode switch selected by the operator, a second buffer circuit U3 to which the luminance of sunrays, measured at the rotation angle by the solar cell sensor unit 300 by the selection of the Manual/Automatic mode switch, is input, a microcomputer U1 receiving the rotation angles of the first and second buffer circuits and the luminance of sunrays to output a rotation angle control signal through Darlington transistors U7 and U8 to the solar cell sensor unit 300 and outputting an operating angle control signal with respect to a maximal solar azimuth calculated by comparing the solar azimuth with respect to the luminance of sunrays with a solar azimuth that is measured in real time in accordance with the period of the real-time counter, a wireless communication module U9 transmitting the operating angle control signal to the tracking device controllers 70, a memory U6 in Which data of the phase current of the AC single phase and the operating angles of the solar module assemblies received from the tracking device controllers 70 through the Wireless communication module U9 are stored, and a TCP/IP communication module U11 transmitting the phase current and operating angle data read and outputted from the memory U6 by the microcomputer U1.The integrated control panel 500 further includes a second switch as a horizontal position angle correction mode switch SW2 correcting initial position angles of the solar module assemblies and a third switch as a time setting mode switch SW3 for real time setting in addition to the first switch as the Manual/ Automatic mode selecting switch SW1. Selecting signals of the first, second, and third switches attached to the outer side of the case are inputted to the microcomputer U1 via a connector W2.The horizontal position angle correction mode switch SW2 is used to initially set horizontal angles of the solar module after the rotation angle sensor 330 is installed to the solar cell sensor unit 300 . In this case, the rotation angle sensor 330 has the same capacity as that of the operating angle sensor 59 of the operating angle sensor unit 50 to output absolutely same rotation angles with respect to displacement. That is, the rotation angle sensor 330 sets a solar cell rotation angle of the solar cell sensor unit 300 to be equal to an operating angle of the operating angle sensor unit 50. The time setting mode switch SW3 sets the time of the integrated panel 500 in an initial state. Signals of the first, second, and third switches, SW1, SW2, SW3, are supplied to the microcomputer U1 through a pull-up resistor. AC electric power of $220 \mathrm{~V}$ is inputted through the connector $\mathrm{W} 1$ and is converted into smoothed DC power by means of a transformer TS1 and bridge diodes U15. In order to supply electric power suitable for driving respective circuits, electric power of $+15 \mathrm{~V}$ DC and $+5 \mathrm{~V}$ DC is supplied to the stepping motor 320 and the microcomputer U1 through a $+15 \mathrm{VDC}$ regulator $\mathrm{U} 13$ and a $+5 \mathrm{VDC}$ regulator U14, respectively. The electric power is supplied to the wireless communication module U9 and the TCP/IP communication module $\mathrm{U} 11$ by a $+3.6 \mathrm{VDC}$ regulator $\mathrm{U} 12$. In order to control the rotation angle of the stepping motor320, the microcomputer $\mathrm{U} 1$ outputs the rotation angle control signal to the stepping motor 320 of the solar cell sensor unit300 using the Darlington transistors U7 
and U8 and connectors W6 and W7. Moreover, an analog input signal is inputted to an analog-digital converter of the microcomputer U1 to provide a function of measuring an environmental factor.

In other Words, in order to detect the rotation angle of the solar cell as the analog input signal, the rotation angle sensor 330 is connected to the connector W3 such that the first buffer circuit $\mathrm{U} 2$ performs the signal processing of the rotation angle, the solar cell output signal as the analog input signal is supplied to the second buffer circuit U3 via a connector W4 such that the second buffer circuit U3 performs a signal processing of luminance, and in order to protect the solar module assemblies from typhoon, an output signal of an anemometer as an analog input signal is supplied to the third buffer circuit U4 via a connector W5 such that the third buffer circuit U4 performs signal processing of Wind velocity. Moreover, an oscillator U16 applies a clock signal to the microcomputer $\mathrm{U} 1$ [1].

The Wireless communication module receives an optimal solar azimuth, transmits the operating angle control signal and a control signal for maintaining the solar module assemblies in a horizontal state when a natural calamity such as a typhoon or a fire is generated, and receives the operating angle of the solar module assemblies and an operation state of the AC single phase inductor.

The Wireless communication module U9 is an RS232C module and is interfaced by the TCP/IP communication module U11 and an interface IC U10. The RS232C, that is, the wireless communication module U9, is connected to the operator's computer via a connector W8 such that the operator remotely monitors the operational state of the integrated control panel. The operator controls the integrated control panel 500 from a long distance using the TCP/IP communication module U11 interfaced with the RS23C module U9 and the interface IC U10.The phase current of the AC single phase inductor and information on the operating angles of the solar module assemblies are stored in the memory U6 such that data stored in the memory U6 are read to be transmitted when the remote monitoring is performed.

The Figure 8(c) shows the circuit diagram illustrating the tracking device controllers that implement the operation angle servo -control. Each of the tracking device controllers includes: a manual/automatic mode switch $\mathrm{S} 1$ selected by an operator; a buffer circuit $\mathrm{J} 2$ to Which the operating angle of the solar module assembly detected by the operating angle sensor unit is inputted when the automatic mode of the manual/automatic mode switch S1 is selected by the operator; wireless communication modules J5 and J6 Which receive an operating angle control signal remotely transmitted by the integrated control panel; a microcomputer $\mathrm{J} 1$ having a memory storing the operating angle and the phase current of the AC single phase inductor and outputting a servo-control signal for the PID servo-control of the solar module assembly to the AC single phase inductor of the high torque driving unit 20, through a forward relay $\mathrm{K} 1$ and a reverse relay $\mathrm{K} 2$ in accordance with a value calculated from the operating angle inputted through the buffer circuit $\mathrm{J} 2$ and the operating angle control signal inputted through the Wireless communication modules J5 and J6, to control the operating angle of the solar module assembly; and an RF communication module or a Bluetooth communication module $\mathrm{J} 7$ remotely transmitting data on the operating angle and the phase current of the AC single phase inductor stored in the memory. In addition to the first switch, the manual/ automatic mode switch S1, there is a second switch, the horizontal position, angle correction mode switch S2 correcting an initial position angle of the solar module assembly, and a third switch, the time setting mode switch S3 for real time setting. Selection signals of the first, second, and third switches attached to the outer side of the case are inputted to the microcomputer J1through a connector P5. The horizontal position angle correction mode switch S2 is used to initially set a horizontal angle of the solar module assembly after the operating angle sensor 59 of the solar module assembly is installed to the high torque driving unit 20. The time setting mode switch S3 sets time of the tracking device controller 70 in an 
initial state. Signals of the first, second, and third switches S1, S2, and S3 are supplied to the forward relay $\mathrm{K} 1$ and the reverse relay $\mathrm{K} 2$ via the connector P1, to be connected to the $\mathrm{AC}$ single phase inductor through the connector P4. In addition, a relay driving IC J4 is used to excite the relays. AC electric power of $220 \mathrm{~V}$ is converted into smoothed DC power by means of a transformer TS2 and bridge diodes $\mathrm{J} 11$. Electric power of $+15 \mathrm{~V}$ DC and $+5 \mathrm{~V}$ DC are supplied to the buffer circuit $\mathrm{J} 2$ and the microcomputer J1 through a $+15 \mathrm{~V}$ DC regulator $\mathrm{J} 8$ and a $+5 \mathrm{VDC}$ regulator $\mathrm{J} 9$, respectively. The electric power is supplied to the Wireless communication modules J5 and J6 and the RF communication module or the Bluetooth communication module $\mathrm{J} 7$ by a $+3.6 \mathrm{~V}$ DC regulator $\mathrm{J} 10$. The Wireless communication modules J5 and J6 include an RS232C module J5 and an RS232C module J6 and are interfaced with the RF communication module or the Bluetooth communication module $\mathrm{J} 7$ and an interface IC J13. The RS232C modules J5 and $\mathrm{J} 6$, the Wireless communication modules, are connected to an operator's computer via a connector $\mathrm{P} 2$ such that the operator remotely monitors the operational state of the tracking device controller. The operator controls the tracking device controller 70 from a distance using the RF communication module or the Bluetooth communication module $\mathrm{J} 7$ interfaced With the RS23C modules J5 and J6 and the interface IC J13. When the AC single phase inductor 170 is continuously operated due to malfunction of the solar module assembly, a signal inputted through a current sensor connector P3 is used by the buffer circuit $\mathrm{J} 2$ to detect the phase current of the AC single phase inductor 170 and to compare an operating time with the reference phase current to determine whether the solar module assembly has malfunctioned on the other hand, in order to verify the precision and reliability of the operating angle control signal remotely received and inputted from the integrated control panel 500 through the wireless communication modules $\mathrm{J} 5$ and $\mathrm{J} 6$, the tracking device controller 70 includes a real time counter (BS1307) J3 by which a period for measuring the solar azimuth by time period in real time can be measured. The microcomputer $\mathrm{U} 1$ of the integrated control panel 500 remotely outputs the operating angle control signal (with respect to the maximal solar azimuth) and the maximal solar azimuth to the tracking device controllers 70 through the wireless communication module U9. Each of the microcomputers $\mathrm{J} 1$ of the tracking device controllers 70 compares the maximal solar azimuth remotely received through the wireless communication modules J5 and J6 with the solar azimuth calculated by a real time azimuth equation using the real time counter (DS1307) J3 and outputs only the verified operating angle control signal to the AC single phase inductors 21 of the high torque driving units 20 [1].

It is illustrating the operating angle servo-control of assemblies to control the operating angles of the solar module assemblies. The operating angle control signal 110 is received from integrated control panel 500 and operating angle output signals 220 of solar module assemblies detected and amplified by angle sensor 210 and compared by operating angle processor 120, hence error of assemblies are compensated by a PID servo controller 130 and supplied to $\mathrm{AC}$ single phase inductor 170, the output operating angle of solar modules is obtained from the contact B of relay 180. The phase current of AC single phase inductor 170 detected by current sensor 160 is compared with reference phase current 230. When the phase current is greater than reference phase current, the power supplied to the AC single phase inductor 170, that is driving the solar modules is interrupted with the help of relay 180, switched from contact B to contact A as shown in Figure 8(b).

The chart Figure 9 describes the remote monitoring and remote control between integrated control panel that can measures in the range of 30 degrees to 150 degrees and tracking device controllers as follows: the luminance of sunrays is measured by the solar sensor unit 300 is analyzed according to the operating angle detected by the operating angle sensor to set the operating angle range for maximum luminance. And this range is compared with the solar azimuth that comes from the solar azimuth equation (820) to calculate the operating angle control signal for solar modules and it is transmitted to the tracking device controllers 70 through wireless communication module [1]. As the 
tracking device controller 70 receives the operating angle control signal from integrated control panel 500, any error of operating angle measured by sensor unit 50 is compensated by PID servo control with the help of calculation 910 to drive the AC single phase inductor 920 that results in driving the solar module assembly 930, by this servo position angle control is implemented. If any malfunction occurs i.e. assembly is unable to track with respect to the operating angle control signal, then tracking device controller 70 transmits both the operating angle of the solar module assembly and the phase current signal of AC single phase inductor to the integrated control panel with wireless link 960. Hence the operator can check the malfunctions by remote monitoring through TCP/IP communication module and can take necessary measures.

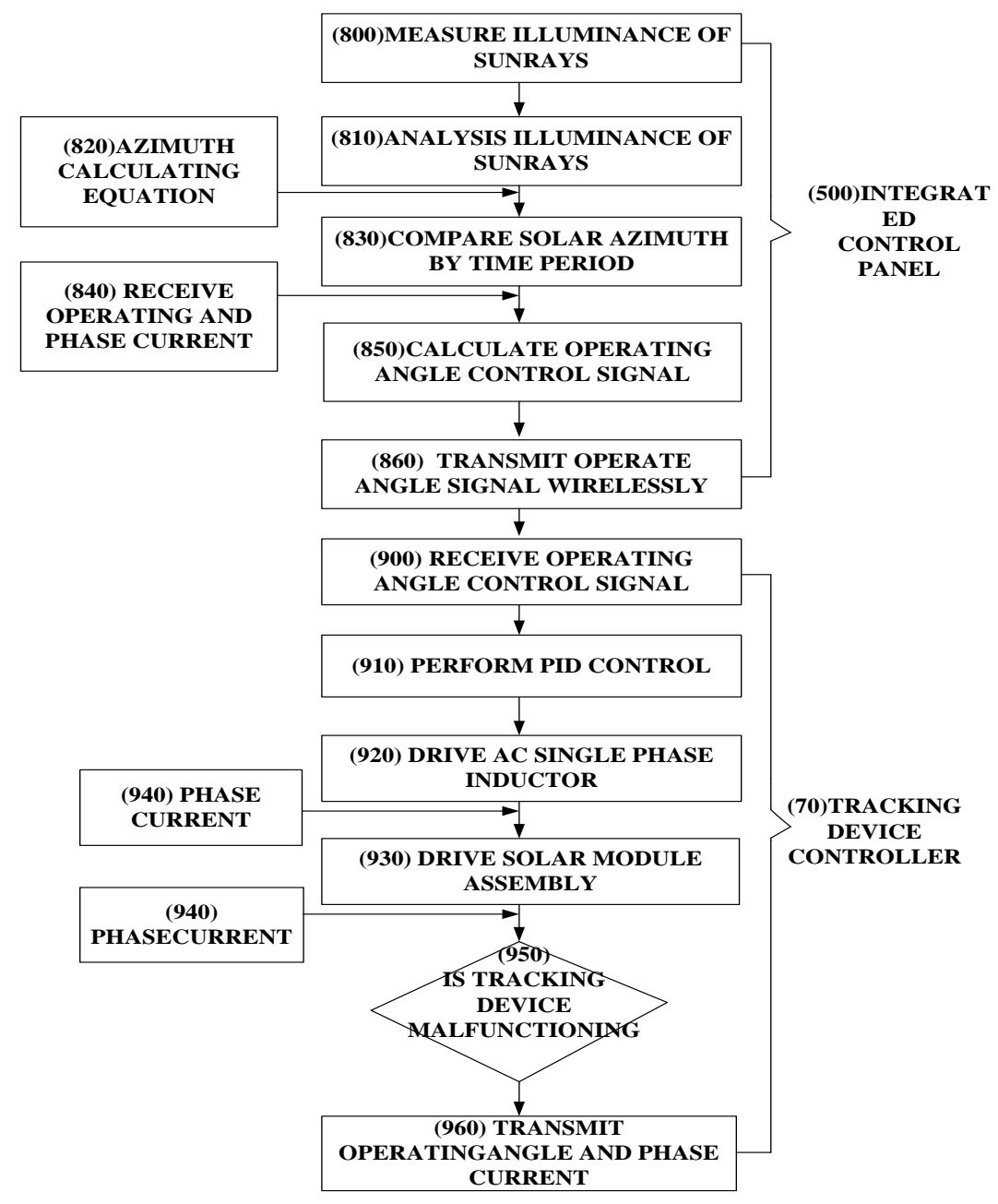

Figure 9. Flow Chart Illustrating the Process of Integrated Control Panel and Tracking Device Controllers 


\section{Experimental Results}

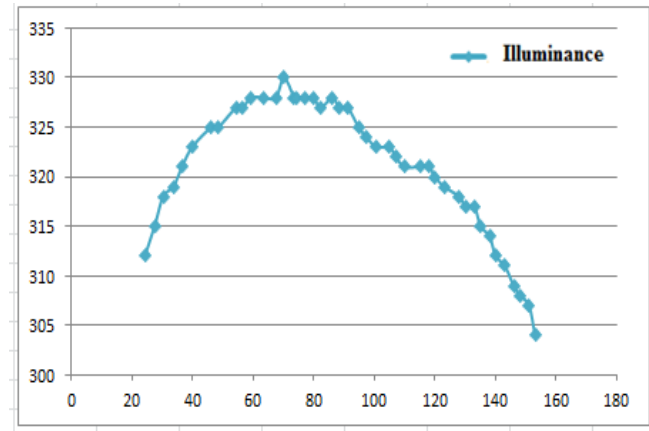

(a)

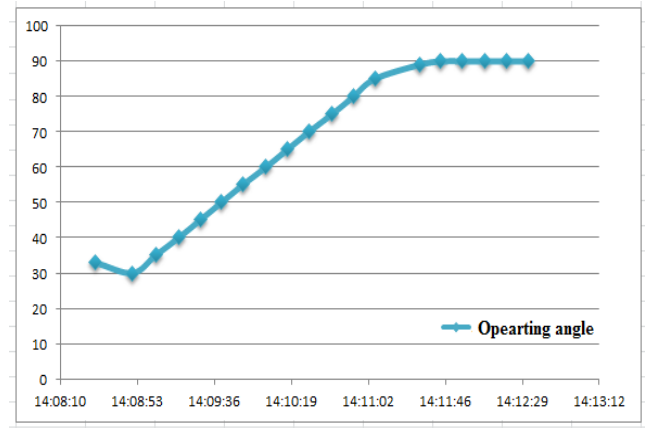

(b)

Figure 10. (A) Characteristics of Solar Cell Sensor Unit (X-Axis Represents A Solar Azimuth and Y-Axis Represents Luminance) (B) Time Response Operation Characteristics of Solar Module Assembly (X-Axis Represents Time and Y-Axis Represents Driving Angle of Solar Module Assembly)

1.The remote control and remote monitoring done between integrated control panel and tracking device controller, the former measures the luminance of sun rays according to the operating angle detected by operating sensor unit, while the later measures the AC single inductor voltage to send the control signals to solar module assemblies.

2. The characteristic curve of solar cell unit as shown in the Figure 10(a). However from the characteristic curve of luminance the solar azimuth is obtained in the range of 24.3 degrees to 153 degrees is observed.

3. As the initial position of 30 degrees to 90 degrees was applied to the solar module assemblies the time response operation characteristics is observed.

4. The manual/automatic mode selectors are installed in the mode selector. The mode selector is switched to the manual mode so that driving signals are supplied to the AC single phase inductor to drive the inductor and to control the operation angles of solar module assemblies.

5. Able to check the malfunctioning of solar module assemblies and can take emergency measures to track the solar luminance. The time response operation characteristics of solar module as shown in the Figure 10(b). The experimental analysis of the one axis solar tracking system efficiency has been improved 5\% to $10 \%$.

\section{Conclusion}

In this paper, the one axis solar tracking system only designed for the vertical aquarium. The one axis solar tracking system gives sufficient power to run the vertical aquarium. From the result, it can be concluded that the precise operating angle servo-control is implemented by using operating angle sensor unit. The each of the tracking device controllers receives optimal signal from the integrated control panel to perform the precise operating angle servo-control efficiency of photo voltaic power generation can be improved by $5 \%$ to $10 \%$. The experimental result of this one axis solar tracking system has been satisfied.

\section{Acknowledgment}

This work supports the KOREA Ministry of Trade, Industry and Energy. We are established the project which is industrial verification and design of ICT VAEMS practical models. Also, this work granted to "Busan University of Foreign Studies". 


\section{References}

[1] K. H. Choi, I.C. Lim, H. W. Kim, H. J. Woo and K. J. Shin, "Solar servo control tracking device", United States Patent No: US 8,042,534 B2,Oct. 25, (2011).

[2] L. Miloudi, "Solar tracking with Photovoltaic Panel", Science Direct, Energy procedia, vol. 42, (2013), pp.103-112.

[3] P.Papageorgas, "Smart solar panels: In-situ monitoring of photovoltaic panels based on wired and wireless sensor networks",Science Direct,Energy Procedia, 36, (2013), pp.535-545.

[4] B. Hanus, "Solar Dachanlagen", Franzis Energie technik, (2009).

[5] Y. Kumsuwan, W. Srirattanawichaikul and S. Premrudeepreechacharn, "Analysis of a two-phase induction motor using dynamic model based on Matlab /Simulink”, (2010).

[6] D. H. Jang and J. S. Won, "Voltage, Frequency, and Phase-Difference Angle Control of PWM Inverters-Fed Two-Phase Induction Motors", IEEE Transactions on Power Electronics, vol. 9, no. 4, (1994), pp. 377-383.

[7] F. Blaabjerg, F. Lungeanu, K.Skaug and M. Tonnes, "Evaluation of Low-Cost Topologies for Two Phase Induction Motor Drives, in Industrial Application", Industry Applications Conference, (2002).

[8] K. Shin, "Realization of solar servo tracking system using the 2 axes solar sensor module", vol 38, no.01, (2015), pp. 1508-1511. 
International Journal of Control and Automation

Vol. 9, No. 10 (2016) 